\title{
Egocentrism and Automatic Perspective Taking in Children and Adults
}

\author{
Andrew D. R. Surtees and Ian A. Apperly \\ University of Birmingham
}

\begin{abstract}
Children (aged 6-10) and adults (total $N=136$ ) completed a novel visual perspective-taking task that allowed quantitative comparisons across age groups. All age groups found it harder to judge the other person's perspective when it differed from their own. This egocentric interference did not decrease with age, even though, overall, performance improved. In addition, it was more difficult to judge one's own perspective when it differed from that of the other person, suggesting that the other's perspective was processed even though it interfered with self-perspective judgments. In a logically equivalent, nonsocial task, the same degree of interference was not observed. These findings are discussed in relation to recent findings suggesting precocious theory-of-mind abilities in infancy.
\end{abstract}

Piaget and Inhelder (1956) suggested that children as old as 7 years found it difficult to judge how a three-dimensional model of three mountains would appear to someone who viewed it from a different angle. Since this classic work there has been a steady downward trend in the age at which children have been shown to have such abilities. Researchers in the 1980s found success on conceptually similar problems in children aged $4-5$ years (Flavell, Everett, Croft, \& Flavell, 1981; Light \& Nix, 1983). Recently, success has been reported on very simple tasks in children as young as 2 years (Moll \& Tomasello, 2006), and perhaps even younger infants (Sodian, Thoermer, \& Metz, 2007; Song \& Baillargeon, 2008). In contrast, we know little about development once children first pass these tasks. This oversight leaves us ignorant about the cognitive characteristics of perspective taking, and whether these change as children gain practice and cognitive resources. We developed a novel task to examine simple perspective taking in children aged from 6 to 10 years and adults.

\section{Egocentrism (Interference From Self-Perspective)}

A recurrent characteristic in young children's explicit perspective-taking judgments is egocentrism: Children's errors show a systematic bias toward the child's own point of view (Fishbein,

The authors would like to thank Jessica Wang and Dana Samson for comments on an earlier draft of this article.

Correspondence concerning this article should be addressed to Andrew D. R. Surtees, School of Psychology, University of Birmingham, Edgbaston, Birmingham, B15 2TT, UK. Electronic mail may be sent to a.surtees@bham.ac.uk.
Lewis, \& Keiffer, 1972; Flavell et al., 1981; Liben, 1978; Piaget \& Inhelder, 1956). Egocentric interference from self-perspective is also observed when older children and adults perform perspectivetaking tasks that are more complex or that require judgments of certainty about what someone else will do or think, with some evidence suggesting that this egocentrism decreases with age (e.g., Bernstein, Atance, Loftus, \& Meltzoff, 2004; Birch \& Bloom, 2004; Epley, Morewedge, \& Keysar, 2004; Keysar, Lin, \& Barr, 2003; Nickerson, 1999; Royzman, Cassidy, \& Baron, 2003). However, although at a general level "egocentrism" may be a recurrent feature of perspective taking, no study has demonstrated egocentrism in older children and adults on tasks equivalent to those used with young children. Thus, in the current work, we tested the degree of egocentrism in children and adults on a Level 1 perspective-taking task that required judgments that are logically equivalent to those required in tests used with children aged 2 and younger (e.g., Flavell et al., 1981).

Samson, Apperly, Braithwaite, and Andrews (2010) and Qureshi, Apperly, and Samson (2010) used a computer-based task in which adult participants made time-pressured judgments about the number of dots seen by a cartoon avatar on the walls of a room. Sometimes the cartoon avatar saw exactly the same number of dots as the participant, and sometimes she or he saw fewer. This is a Level

(C) 2012 The Authors

Child Development (C) 2012 Society for Research in Child Development, Inc. All rights reserved. 0009-3920/2012/8302-0007

DOI: $10.1111 /$ j.1467-8624.2011.01730.x 
1 perspective-taking task because it requires judgments about what someone sees, whereas a Level 2 perspective-taking task requires judgments about the particular way in which they see something (Flavell et al., 1981). When they judged the avatar's perspective, participants were slower and more error prone if they saw more dots than the avatar. This is evidence of egocentric bias because it shows that participants' own discrepant perspective interfered with judgments of the avatar's perspective. We adapted this task in order to compare the size of the egocentric effect observed in adults with the effect observed in children aged 6-10 years.

\section{Automaticity}

Although Samson et al. (2010) found that participants' own perspective interfered with their explicit judgments about the avatar's perspective, the same series of experiments provided evidence that the avatar's perspective itself was being calculated in a relatively automatic manner. One signature of an automatic process is that it may be executed even when this interferes with successful performance on some other task (Dijkerman \& Smit, 2007; Kilner, Paulignan, \& Blakemore, 2003; Lefevre, Bisanz, \& Mrkonjic, 1988). To test for this, Samson et al.'s experiments also included "self" trials where participants judged how many dots they themselves could see on the wall of the room. The important finding was that these self-perspective judgments were slower and more error prone when the avatar saw fewer dots than the participant. This effect indicated that even though the avatar's perspective was irrelevant and, in principle, could be ignored on self-perspective trials, in fact the avatar's perspective was processed sufficiently that this resulted in interference when participants had to judge their own perspective. A further experiment in Samson et al.'s study found that this effect persisted even when participants only ever made judgments about their own perspective for the entire experiment. That is to say, participants appeared to be processing the avatar's perspective even when it was wholly irrelevant to the task at hand, and this interfered with participants' judgments about their own perspective when their own perspective was different from that of the avatar. To this extent, these data show evidence of automatic Level 1 perspective processing in adults.

There are at least two ways in which adults might come to calculate other people's perspectives in an automatic manner. One possibility is that Level 1 perspective taking is not initially automatic in children, but by adulthood repeated practice has resulted in automatization. If so then, on Samson et al.'s (2010) task, younger children should not process the avatar's perspective automatically, and so should suffer less or even no interference from the avatar's perspective when they judge their own self-perspective. Another possibility is that the automaticity observed in adults reflects the operation of a cognitively efficient process for simple perspective taking that has been present since infancy. This possibility is consistent with evidence that infants and young children may show precocious sensitivity to the perspectives of others when tested indirectly (via looking times or eye movements) rather than when required to make an explicit judgment (e.g., Clements \& Perner, 1994; Onishi \& Bailargeon, 2005; Sodian et al., 2007; Song \& Baillargeon, 2008; Southgate, Senju, \& Csibra, 2007). Such "original automaticity" predicts that the interference effects observed in adults by Samson et al. (2010) will be observed in children of all ages, if only appropriate methods could be found to test for these effects. In the current study, we sought to make some progress on this question in Experiment $1 \mathrm{~A}$ by testing children as young as 6 years on a paradigm very similar to that used by Samson et al. In Experiment 1B, we tested whether similar effects would be observed in an analogous task that did not require perspective taking.

\section{Experiment 1A}

\section{Method}

Overview. On every trial participants heard instructions and viewed a cartoon avatar standing in a cartoon room with dots on the wall (see Figure 1). On self trials, participants judged the number of dots they could see on the walls of a room. On other trials, participants judged how many dots could be seen by the cartoon avatar in the picture. On consistent trials the avatar could see the same number of dots as the participant. On inconsistent trials the avatar's position in the room meant that she or he saw fewer dots. Although it would have been interesting to create a situation in which it was the avatar that saw the greater number of dots, the current paradigm does not permit such a scenario as the only way to ascertain how many dots she or he sees is for participants to see at least as many for themselves.

Participants. Four groups of participants were tested: undergraduates $(N=11)$, mean age $=19.1$ (range $=18-23), 10$ female; 10 -year-olds $(N=24)$, 


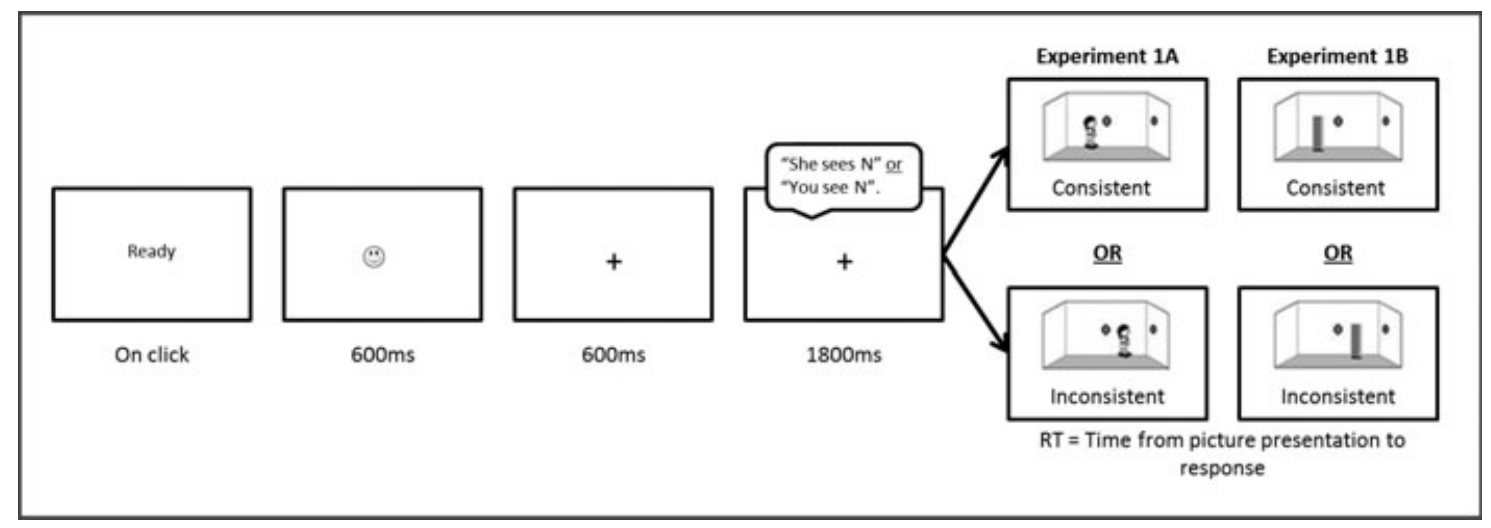

Figure 1. Schematic event sequence of experimental trials.

Note. Participants were cued as to the perspective they were to take and the number to verify. Following this they were presented with a picture stimulus. For Experiment 1B, "She sees $N^{\prime \prime}$ was replaced by "yellow-side $N$. ."

mean age $=10.1 \quad($ range $=9 ; 6-10 ; 5), 13$ female; 8 year-olds $(N=35)$, mean age $=8.3$ years (range $=$ $8 ; 0-8 ; 5), \quad 18$ female; 6-year-olds $(N=36)$, mean age $=6.4$ (range $=6 ; 1-6 ; 11)$, 20 female. Adult participants were recruited for course credits, and were predominantly White British. Child participants were recruited from two schools in a working middle-class area of Wolverhampton; as for adults, the majority of participants were White British.

Design and procedure. Child participants completed the experiment in a room adjacent to their normal classroom. Adult participants completed the task in a testing cubicle. Instructions included a detailed description of the procedure and an instruction to respond as quickly and accurately as possible. Practice trials were completed until the participant had successfully answered a question for each of the four conditions (self-consistent, selfinconsistent, other-consistent, other-inconsistent). These practice trials were systematically blocked into groups of four trials (one for each condition). No participant required more than two presentations of any condition to achieve success.

On each trial, participants viewed successive fixation stimuli (a smiling face [600 ms] and a fixation cross [600 ms]) followed by a 1,800-ms auditory stimulus (either "He sees $N$ " or "You see $N$," where $N$ ranged from 1 to 3 , so that the number of dots was within the range that could be enumerated quickly and accurately via subitization; Trick \& Pylyshyn, 1994) and then the test picture depicting an avatar in a room with 1-3 dots on the wall. Participants pressed one of two colored keys to indicate whether or not the auditory stimulus correctly described the picture (on half of the trials, the auditory stimulus did match the picture, and on half it did not). Because the cartoon character either saw the same number of dots as the participant, or fewer dots, it was not possible to match self and other trials for both the number of dots in the picture stimuli and the numbers described in the sentence stimuli. For the present experiments, we matched the sentence stimuli across self and other trials, since we thought that systematic differences in the sentence stimuli were more likely to have unintended effects on children's performance than differences in the picture stimuli. For this reason, we should be somewhat cautious in interpreting any overall difference between self and other conditions, but importantly, this has no effect on the critical comparisons between consistent and inconsistent trials, which were made within self and other conditions.

Response time (RT) was measured from the onset of the picture. Participants completed four practice trials. Child participants completed 48 test trials: 24 where self and other perspectives were consistent (12 self, 12 other) and 24 where self and other perspectives were inconsistent (12 self, 12 other). Adults completed two cycles of a child experiment (96 trials). Self and other trials were pseudorandomly mixed within each block of trials (no block contained more than three trials in a row without a change in consistency, perspective, response button, and direction of avatar). The experiment was presented using E-Prime 2.0 (Schneider, Eschmann, \& Zuccolotto, 2002) on a laptop computer.

\section{Results and Discussion}

Examining the effect of perspective consistency on Other trials allowed us to test for egocentric interference from self-perspective when making 
explicit judgments about the avatar's perspective. Examining the effect of perspective consistency on self trials allowed us to test whether the avatar's perspective was processed automatically, leading to interference with judgments about self-perspective. Only trials where the auditory stimulus and the picture matched were used in the analysis. Following Samson et al. (2010) and Qureshi et al. (2010), nonmatched trials were excluded due to systematic differences between consistent and inconsistent conditions on these trials. Specifically, for inconsistent trials, the number participants had to reject was the content of the other perspective in the scene, whereas for consistent trials it was a random number less than four. Rejecting a stimulus linked to another possible response could, in itself be more difficult than rejecting a stimulus not linked to another response. Also, for self-perspective-taking nonmatched inconsistent trials involved rejecting a number that was systematically smaller than for consistent trials. These design features for nonmatched trials were an unavoidable consequence of the task design but did not affect trials where the sentence and picture matched. Mean RT and error proportion data for both Experiments $1 \mathrm{~A}$ and $1 \mathrm{~B}$ are presented in Figure 2.

Response times. Data that were $2 S D$ from the mean were omitted from the analysis of RTs $(3.6 \%$ of the data for 6-year-olds, $2.9 \%$ for 8 -year-olds,
$4.3 \%$ for 10 -year-olds, and $2.1 \%$ for adults), as were data from incorrect responses.

An analysis of variance (ANOVA) with perspective and consistency as within-subjects factors, and age group as a between-subjects factor revealed an effect of age, $F(3,105)=81.94, p<.001, \eta_{p}{ }^{2}=.709$. Two-tailed $t$ tests showed that each older age group responded more quickly than the adjacent younger age group, lowest $t(58)=6.16$, all $p s<.001$. There was a main effect of perspective, $F(1,105)=10.558$, $p=.002$, self $<$ other, and a main effect of consistency, $F(1,105)=32.423, p<.001, \eta_{p}{ }^{2}=.243$, consistent $<$ inconsistent. There were no significant interactions between variables, $F_{\mathrm{s}} \leq 1.297$, ps $\geq .280$, $\eta_{p}{ }^{2} \mathrm{~s} \leq .037$.

Errors. A similar ANOVA on proportion of errors revealed no main effect of age, $F(3,105)$ $=1.230, p=.303, \eta_{p}{ }^{2}=.107$. There was a main effect of consistency, $F(1,105)=12.467, p<.001$, $\eta_{p}{ }^{2}=.108$, consistent < inconsistent, but no effect of perspective, $F(1,105)=2.206, p=.141, \eta_{p}{ }^{2}=$ .021. There was a significant three-way interaction between perspective, consistency, and age, $F(3$, $105)=3.95, p=.01, \eta_{p}{ }^{2}=.10$. There were no other significant interactions, $F \mathrm{~s} \leq 1.968, \quad p s \geq .123$, $\eta_{p}^{2} \mathrm{~s} \leq .054$.

The three-way interaction was followed up with 2 (perspective) $\times 2$ (consistency) ANOVAs for each age group and where significant interactions were
OTHER
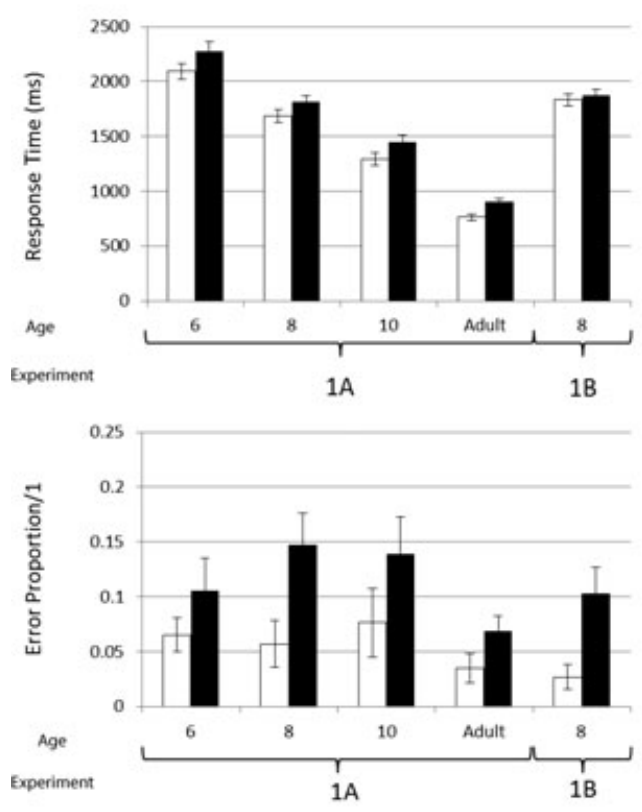

SELF
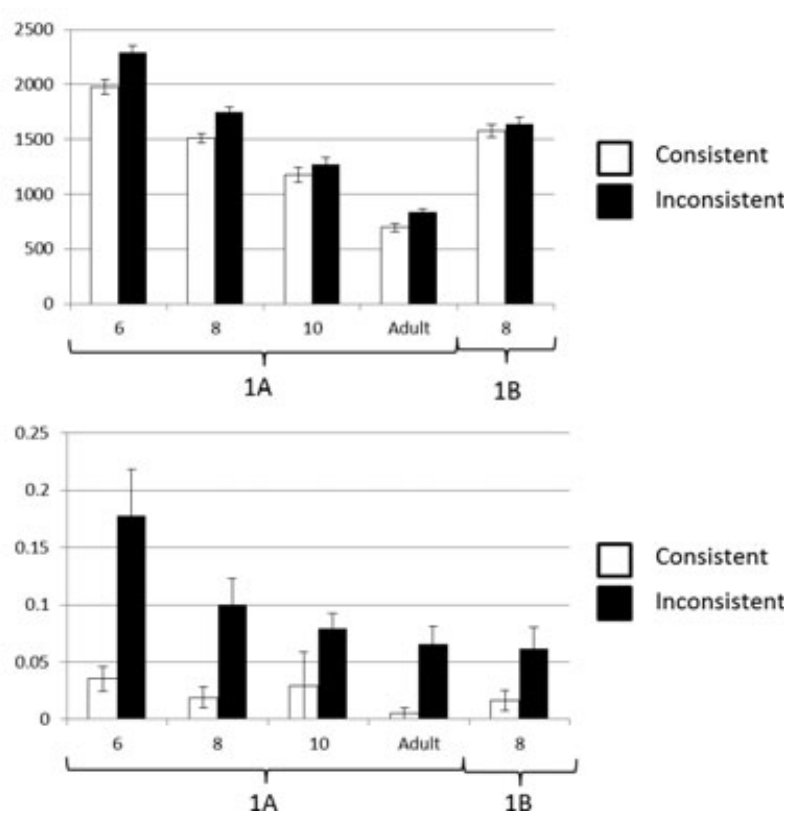

Figure 2. Mean response times and error proportions of children and adults in Experiments 1A and 1B. Note. Error bars indicate standard errors of the mean. 
found, relevant two-tailed $t$ tests. For 6-year-olds there was a significant interaction between consistency and perspective, $F(1,37)=5.952, p=.02$, $\eta_{p}{ }^{2}=.139$, with an effect of consistency for self $(t(37)=3.283, \quad p=.002$, inconsistent $>$ consistent, but not for other, $t(37)=1.308, p=.199$, trials. For 8 -year-olds there was no significant interaction between consistency and perspective, $F(1,34)$ $=0.051, p=.822, \eta_{p}{ }^{2}=.002$. For 10-year-olds there was a significant interaction between consistency and perspective, $F(1,23)=4.666, p=.041$, $\eta_{p}^{2}=.169$, with an effect of consistency for other, $t(23)=1.696, p=.103$, but not for self, $t(23)=1.519$, $p=.142$, trials. For adults there was a significant interaction between consistency and perspective, $F(1,10)=4.666, p=.041, \eta_{p}^{2}=.169$, with an effect of consistency for self, $t(10)=3.23, p=.01$, but not for other, $t(10)=1.56, p=.15$, trials. Overall, the three-way interaction between age, perspective, and consistency is somewhat difficult to interpret, with the form of the interaction varying between age groups but not changing systematically with increasing age. Notably, for all ages and for both self and other conditions, the effects of consistency are either significant or in the same direction as the significant effects (i.e., more errors on inconsistent trials), reassuring us that the results from the RT analysis are not due to speed-accuracy trade-offs.

Experiment 1A clearly showed that participants were processing both perspectives, even when it was not necessary for the task and, in cases where perspectives were inconsistent, when it actively hindered performance. However, using a mixedblock design, it is possible that the interference caused was due to executive demands on task switching or due to participants adopting a strategy that took into account both possible options for each trial. Experiment $1 \mathrm{~B}$ addressed this with a task that matched the one used in Experiment $1 \mathrm{~A}$ in terms of the possibility of task-switching costs or the adoption of strategies, but which did not entail perspective taking.

\section{Experiment 1B}

To test the specificity of the effects found in Experiment $1 \mathrm{~A}$ to the social domain, we tested a further sample of 8-year-old children using nonsocial stimuli. Only one age group was tested as all age groups had shown qualitatively similar performance in Experiment 1A. Eight-year-olds were selected as they formed the midpoint of our child age group.

\section{Method}

Participants. In Experiment 1B, 30 participants (mean age $=8.2$; range $=8 ; 0-8 ; 5,18$ female) were tested. Participants were recruited from a school in a working middle-class area of Wolverhampton; the majority of participants were White British.

Design and procedure. Experiment $1 \mathrm{~B}$ was identical to Experiment 1A except that a yellow and blue stick replaced the cartoon child, and the verbal cue "yellow-side $N$ " replaced the verbal cue "she or he sees N." Participants judged whether the number, $N$, was correct for the number of dots on the yellow side of the stick. Thus, the task was structurally similar to that in Experiment 1A, but had no social stimulus.

\section{Results and Discussion}

For each analysis, we conducted an ANOVA to investigate the effect of perspective and consistency solely in Experiment 1B. Following this, a Perspective $\times$ Consistency $\times$ Experiment ANOVA was completed to compare results from Experiment 1B with results from the 8-year-olds in Experiment 1A. Within-experiment ANOVAs evaluate interference from purely arbitrary task demands, while betweenexperiment ANOVAs test whether the patterns observed in Experiment 1A with social stimuli were significantly different from those observed in Experiment 1B with nonsocial stimuli.

Response times. Outliers (3.2\% of the data set) were removed using the same criterion as in Experiment $1 \mathrm{~A}$.

An ANOVA to investigate the effects of perspective and consistency on RTs in Experiment $1 \mathrm{~B}$ revealed a main effect of perspective, $F(1,30)$ $=28.388, p<.001, \eta_{p}{ }^{2}=.486$, self $<$ stick, but no main effect of consistency, $F(1,30)=2.363, p=.135$, $\eta_{p}{ }^{2}=.073$, and no significant interaction between perspective and consistency, $F(1,30)=0.256, p=$ $.617, \eta_{p}{ }^{2}=.008$.

An ANOVA with perspective and consistency as within-subjects factors and experiment as a between-subjects factor revealed a main effect of perspective, $F(1,64)=39.401, p<.001, \eta_{p}{ }^{2}=.381$; an effect of consistency, $F(1,64)=17.054, p<.001$, $\eta_{p}^{2}=.210$; but not an effect of Experiment, $F(1$, 64) $=0.763, p=.386, \eta_{p}{ }^{2}=.012$. There was not a significant interaction between perspective and consistency, $F(1,64)=2.097, p=.152, \eta_{p}^{2}=.007$, but there were interactions between perspective and experiment, $F(1,64)=4.758, p=.033, \eta_{p}{ }^{2}=.069$, and importantly between experiment and consistency, $F(1,64)=4.126, p=.046, \eta_{p}{ }^{2}=.061$. The 
three-way interaction between experiment, perspective, and consistency was not significant, $F(1$, 64) $=0.432, p=.514, \eta_{p}^{2}=.007$.

The interaction between perspective and experiment highlights that the effect of perspective was greater in Experiment $1 \mathrm{~B}$ than for 8-year-olds in Experiment 1A, $F(1,34)=10.705, p=.002, \eta_{p}{ }^{2}=$ .239 , although this was significant in both cases. The interaction between consistency and experiment highlights that the (significant) effect of consistency for 8-year-olds in Experiment 1A, $F(1$, $34)=18.246, p<.001, \eta_{p}{ }^{2}=.349$, was greater than the (ns) effect of consistency in Experiment 1B.

Errors. An ANOVA to investigate effects of perspective and consistency on the proportion of errors in Experiment 1B revealed a trend toward an effect of perspective, $F(1,30)=3.693, p=.064$, $\eta_{p}{ }^{2}=.110$, self < stick; an effect of consistency, $F(1,30)=11.579, p=.002, \eta_{p}^{2}=.278$, consistent $<$ inconsistent; but no interaction between the two, $F(1,30)=1.054, p=.313, \eta_{p}{ }^{2}=.034$.

An ANOVA with perspective and consistency as within-subjects factors and experiment as a between-subjects factor revealed a main effect of perspective, $F(1,64)=7.546, p=.008, \eta_{p}{ }^{2}=.105$; an effect of consistency, $F(1,64)=28.848, p<.001$, $\eta_{p}{ }^{2}=.311$; but no effect of experiment, $F(1,64)$ $=2.966, p=.090, \eta_{p}{ }^{2}=.044$. There were no significant interactions, $F \mathrm{~s} \leq 0.817, p s \geq .369$.

That we observed an interaction between consistency and experiment when examining RTs indicates that the effects of consistency in Experiment $1 \mathrm{~A}$ arose from interference between self and other perspectives (a demand that could only arise Experiment 1A) and was not due to having to switch between two tasks (a demand that was present in both Experiments 1A and 1B). By the same reasoning, the fact that there was no interaction between experiment and consistency in the analysis of errors suggests that the demand for task switching might have given rise to more errors on inconsistent trials in both Experiments 1A and 1B.

\section{General Discussion}

We measured the speed and accuracy of self and other perspective taking of adults and children on a very simple visual perspective-taking task logically equivalent to those passed by children aged 2 years or younger. At all ages we found evidence of egocentrism, and of the opposite effect of interference from the other's perspective when judging selfperspective.

\section{Self versus Other Perspective Taking}

In Experiment 1A, we found that participants were faster to make judgments about their own perspective than that of an avatar. Experiment $1 \mathrm{~B}$ found that for 8-year-olds at least, there was an even greater difference between fast self judgments compared to slower judgments about the number of dots on the yellow side of the stick. As described in the Method section, comparisons between self and other (or self and stick) must be treated with caution because, while probe sentences were identical in the two conditions, the pictures in other (or stick) trials included a higher proportion of items with two or three dots than self trials. However, since the number of dots for all trials was within the range that participants could enumerate in a fast, parallel process of subitization (Trick \& Pylyshyn, 1994) it seems unlikely that this difference contributed very strongly to the condition differences observed. Therefore, this concern notwithstanding, it is notable that the observation of faster responses in self trials is compatible with the hypothesis that explicit perspective taking is achieved through anchoring and adjustment (Epley et al., 2004). This hypothesis suggests that when perspective-taking people first take their own perspective and then adjust this to calculate another's. It would follow quite naturally that judging self perspective would be faster and more accurate than judging other perspectives. Moreover, the observation that the difference between self and other in Experiment 1A was smaller than the difference between self and stick in Experiment 1B fits with evidence that explicit perspective taking is easier when participants can easily imagine themselves as occupying the body of the alternative position (e.g., Kessler \& Thomson, 2010). Importantly, however, whatever the origin of these effects, it is notable that they apply irrespective of whether self and other trials are consistent with each other. We discuss the effects of perspective consistency in the following sections.

\section{Egocentrism}

Egocentric errors-by which we mean errors that show an influence of one's own privileged perspective-are common when young children perform simple perspective-taking tasks, and egocentric bias is common when adults complete more complex tasks (Bernstein et al., 2004). The current study is the first to show that the egocentrism observed in young children's errors on the very simplest (Level 1) visual perspective-taking tasks is also apparent 
in errors and RTs throughout development into adulthood. This suggests that egocentrism in adults does not merely resemble egocentric phenomena observed in children but, for simple perspective taking at least, reflects the same underlying cognitive processes: We cannot help having our own perspective and this interferes with judgments about the perspective of others. Interestingly, although there was age-related improvement in general speed and accuracy of perspective taking, there was no reduction in the size of the egocentric effect, as demonstrated by the absence of an interaction between age and perspective consistency. This suggests that age-related increases in general processing resources such as inhibitory control may improve the efficiency of perspective taking, but not by reducing egocentrism. We remain relatively neutral as to the nature of the egocentrism operating here. On the one hand, it is clear that this interference is from a self-view, but in all our trials the self-view of the scene may also be considered (in Light \& Nix's, 1983, terms) a "good view." There is potential for further investigation of this by degrading self-perspective, and so generating a self-view that is not a "good view." In Experiment 1B, when RTs were analyzed, we found a significantly smaller effect of the interference from self on a task that did not involve social stimuli, compared to the analogous effect in Experiment 1A. This suggests that there may be cases where egocentrism is most strongly a social phenomenon. However, we did find an overall effect of consistency on accuracy in Experiment 1B, which supports previous findings that tasks do not necessarily have to be social for egocentrism to be found (Perner \& Leekam, 2008).

\section{"Altercentric" Interference from the Avatar's Perspective}

Consistent with Samson et al. (2010) and Qureshi et al. (2010), we found that participants' judgments about their own perspective were slower and more error prone when the avatar in the room had a different perspective. Importantly, the same degree of interference was not observed in RTs in Experiment 1B, where children performed a structurally similar task with nonsocial stimuli. These effects can be explained if we suppose that in Experiment 1A children and adults calculated the number of dots that the avatar could see even when they were not told to but did not (or were less likely to) perform the equivalent calculation of the number of discs on the yellow side of the stick in Experiment 1B. That is to say, if we view the presence of altercentric interference on self trials as an indirect measure of participants' tendency to calculate the avatar's perspective, the results indicate a relatively automatic process of Level 1 perspective calculation in children as well as in adults. In combination with the results from other trials, this finding indicates that participants may automatically process the visual perspective of the avatar, resulting in interference with self judgments, but additional work is necessary to select this information for an explicit judgment of the avatar's perspective on other trials. This analysis is compatible with the findings of Qureshi et al. who found that adults' performance of a secondary executive task at the same time as a perspective-taking task similar to the one used here does not disrupt adults' calculation of perspectives but does disrupt their selection of the appropriate self or other perspective for an explicit judgment.

Of course, it is important to consider why participants in Experiment 1A were processing the avatar's perspective automatically. Our results add to the existing evidence from Samson et al. (2010) in suggesting it is highly unlikely that the effects found in Experiment $1 \mathrm{~A}$ are simply the unintended result of task-switching effects arising from our experimental design. That is to say, from Experiment $1 \mathrm{~A}$ alone it might have been that on self trials participants computed the irrelevant perspective of the avatar (and so suffered interference when it was inconsistent with their own) only because of carryover from the explicit requirement to take the avatar's perspective on other trials of the experiment. The results of Experiment 1B make this interpretation seems highly unlikely, however, because the general requirements on task switching between self and stick trials were just the same in Experiment $1 \mathrm{~B}$ as the requirements on task switching between self and other trials in Experiment 1A, and yet no interference was observed in Experiment $1 \mathrm{~B}$. Thus, the significantly greater interference effects observed in Experiment 1A would seem to be due to the presence of the social stimulus, and not due to the overall design of the task.

The current studies do not make it clear precisely which features of the avatar condition are necessary to drive automatic perspective taking. For example, it is possible that automatic perspective taking will only be driven if the avatar's eyes are visible, or if its head direction or body orientation is visible, or it might be that eye direction, head direction, and body direction all make independent contributions to this effect. Future research may also reveal that participants' beliefs about the avatar may influence their processing in a top-down fashion. For 
example, Teufel et al. (2009) recently found that the tendency for participants to follow the gaze of a face stimulus - a phenomenon that appears highly automatic on many criteria-could be attenuated if participants believed that the face stimulus could not in fact see anything. It is conceivable that participants' "automatic" processing of the avatar's perspective in the paradigm used in the current studies might be overridden with a similar manipulation. Importantly, however, although results from such studies would advance our understanding of the origin of the effects observed here, none of them would alter our principal conclusion that altercentric interference-resulting from relatively automatic perspective taking-is evident in children as well as in adults.

\section{Automatization or Original Automaticity?}

The absence of an age-related change in the size of the interference effect from the avatar's perspective suggests that automatic perspective taking is not altered by increasing practice or availability of cognitive resources, at least in children over the age of 6 . That is to say, the current study found no evidence that adults' automatic perspective calculation is the result of automatization. However, we cannot rule out the possibility that children have already fully automatized the calculation of simple visual perspectives by the age of 6 . It is quite possible that children have had enough exposure to social stimuli to have automatized the process of perspective taking. Pilot work suggested that the current methods were unsuitable for younger children, but testing for similar interference effects between self and other in younger children's self-perspective taking is an important avenue for future investigation.

Although the current data do not rule out automatization, they are clearly compatible with the alternative "original automaticity" hypothesis, whereby children and adults have a cognitively efficient ability for simple perspective taking that is already present in infants. This view gains plausibility from the growing body of evidence that infants and young children show sensitivity to simple perspectives when these abilities are tested indirectly via looking times, eye movements, or spontaneous actions (e.g., Clements \& Perner, 1994; Onishi \& Bailargeon, 2005; Sodian et al., 2007; Song \& Baillargeon, 2008; Southgate et al., 2007). And indeed, the current findings may give insight into why infants and young children fail on more traditional explicit tests of the same abilities. Our findings suggest that information about what an agent sees may be automatically calculated (resulting in self-other interference on self trials) but that making explicit judgments about what the agent sees opens the door to egocentric interference (resulting in selfother interference on other trials). We suggest that adults and older children have the executive resources necessary to resist such egocentric interference, and so their explicit judgments are merely slower when self and other perspectives are in conflict. Infants and young children may lack such executive resources, and so their explicit judgments are dominated by their own perspective (see e.g., Friedman \& Leslie, 2005; Leslie, 1987, 2005).

This clearly raises the question of why explicit judgments require additional cognitive resources, and what purpose such explicit judgments might serve if information about other people's perspectives is already being calculated automatically. One hypothesis is proposed by Apperly and Butterfill (2009), who suppose that any capacities for automatic processing of visual perspective (or, for that matter, other mental states such as beliefs) will be limited to relatively simple cases. As one example of such a limitation, Apperly and Butterfill (2009) and Apperly (2010) suggest that Level 1 perspective taking may be automatic whereas Level 2 perspective taking may not be. On this account, explicit judgments recruit more general reasoning processes, which carry the cost of demands on memory and executive control, but bring the benefit of more flexible perspective taking. Whether or not this particular hypothesis is correct, it is a priority for future work to investigate the role and scope of automatic versus controlled processes in making judgments about the perspectives of others.

\section{References}

Apperly, I. A. (2010). Mindreaders: The cognitive basis of "theory of mind." Hove, UK: Psychology Press.

Apperly, I. A., \& Butterfill, S. A. (2009). Do humans have two systems to track beliefs and belief-like states. Psychological Review, 116, 953-970.

Bernstein, D. M., Atance, C., Loftus, G. R., \& Meltzoff, A. (2004). We saw it all along-Visual hindsight bias in children and adults. Psychological Science, 15, 264-267.

Birch, S. A. J., \& Bloom, P. (2004). Understanding children's and adults' limitations in mental state reasoning. Trends in Cognitive Sciences, 8, 255-260.

Clements, W. A., \& Perner, J. (1994). Implicit understanding of belief. Cognitive Development, 9, 377-395.

Dijkerman, H. C., \& Smit, M. C. (2007). Interference of grasping observation during prehension, a behavioural study. Experimental Brain Research, 176, 387-396. 
Epley, N., Morewedge, C. K., \& Keysar, B. (2004). Perspective taking in children and adults: Equivalent egocentrism but differential correction. Journal of Experimental Social Psychology, 40, 760-768.

Fishbein, H. D., Lewis, S., \& Keiffer, K. (1972). Childrens understanding of spatial relations-Coordination of perspectives. Developmental Psychology, 7, 21-33.

Flavell, J. H., Everett, B. A., Croft, K., \& Flavell, E. R. (1981). Young childrens knowledge about visualperception-Further evidence for the Level 1-Level 2 distinction. Developmental Psychology, 17, 99-103.

Friedman, O., \& Leslie, A. M. (2005). Processing demands in belief-desire reasoning: Inhibition or general difficulty? Developmental Science, 8, 218-225.

Kessler, K., \& Thomson, L. A. (2010). The embodied nature of spatial perspective taking: Embodied transformation versus sensorimotor interference. Cognition, 114, 72-88.

Keysar, B., Lin, S. H., \& Barr, D. J. (2003). Limits on theory of mind use in adults. Cognition, 89, 25-41.

Kilner, J. M., Paulignan, Y., \& Blakemore, S. J. (2003). An interference effect of observed biological movement on action. Current Biology, 13, 522-525.

Lefevre, J. A., Bisanz, J., \& Mrkonjic, L. (1988). Cognitive arithmetic-Evidence for obligatory activation of arithmetic facts. Memory \& Cognition, 16, 45-53.

Leslie, A. M. (1987). Pretense and representation-The origins of theory of mind. Psychological Review, 94, 412-426.

Leslie, A. M. (2005). Developmental parallels in understanding minds and bodies. Trends in Cognitive Sciences, 9, 459-462.

Liben, L. S. (1978). Perspective-taking skills in young-children-Seeing world through rose-colored glasses. Developmental Psychology, 14, 87-92.

Light, P., \& Nix, C. (1983). Own view versus good view in a perspective-taking task. Child Development, 54, 480-483.

Moll, H., \& Tomasello, M. (2006). Level I perspective-taking at 24 months of age. British Journal of Developmental Psychology, 24, 603-613.

Nickerson, R. S. (1999). How we know-and sometimes misjudge-What others know: Imputing one's own knowledge to others. Psychological Bulletin, 125, 737-759.
Onishi, K. H., \& Bailargeon, R. (2005). Do 15-month-old infants understand false beliefs? Science, 308, 255-258.

Perner, J., \& Leekam, S. (2008). The curious incident of the photo that was accused of being false: Issues of domain specificity in development, autism, and brain imaging. Quarterly Journal of Experimental Psychology, 61, 76-89.

Piaget, J., \& Inhelder, B. (1956). The child's conception of space. London: Routledge \& Kegan Paul.

Qureshi, A., Apperly, I. A., \& Samson, D. (2010). Executive function is necessary for perspective-selection, not Level-1 visual perspective-calculation: Evidence from a dual-task study of adults. Cognition, 117, 230-236.

Royzman, E. B., Cassidy, K. W., \& Baron, J. (2003). "I know, you know": Epistemic egocentrism in children and adults. Review of General Psychology, 7, 38-65.

Samson, D., Apperly, I. A., Braithwaite, J. J., \& Andrews, B. A. (2010). Seeing it their way: Evidence for rapid and involuntary computation of what others see. Journal of Experimental Psychology: Human Perception and Performance, 36, 1255-1266.

Schneider, W., Eschmann, A., \& Zuccolotto, A. (2002). EPrime user's guide. Pittsburgh, PA: Psychology Software Tools.

Sodian, B., Thoermer, C., \& Metz, U. (2007). Now I see it but you don't: 14-month-olds can represent another person's visual perspective. Developmental Science, 10, 199-204.

Song, H. J., \& Baillargeon, R. (2008). Infants' reasoning about others' false perceptions. Developmental Psychology, 44, 1789-1795.

Southgate, V., Senju, A., \& Csibra, G. (2007). Action anticipation through attribution of false belief by 2-yearolds. Psychological Science, 18, 587-592.

Teufel, C., Alexis, D. M., Todd, H., Lawrance-Owen, A. J., Clayton, N. S., \& Davis, G. (2009). Social cognition modulates the sensory coding of observed gaze direction. Current Biology, 19, 1274-1277.

Trick, L. M., \& Pylyshyn, Z. W. (1994). Why are small and large numbers enumerated differently-A limitedcapacity preattentive stage in vision. Psychological Review, 101, 80-102. 